\title{
Pathology of Hepato-renal Disorder due to Non-infectious Etiology in Swine
}

\author{
Binita Baruah $^{1 *}$, Sarojini Mahanta Tamuli ${ }^{1}$, Taibur Rahman ${ }^{1}$, Shantanu Tamuly ${ }^{2}$ and Sophia Gogoi ${ }^{3}$ \\ ${ }^{1}$ Department of Veterinary Pathology, College of Veterinary Science, Assam Agricultural University (AAU), Khanapara, \\ Guwahati, Assam, INDIA \\ ${ }^{2}$ Department of Veterinary Biochemistry, College of Veterinary Science, Assam Agricultural University (AAU), Khanapara, \\ Guwahati, Assam, INDIA \\ ${ }^{3}$ Department of Veterinary Microbiology, College of Veterinary Science, Assam Agricultural University (AAU), Khanapara, \\ Guwahati, Assam, INDIA \\ "Corresponding author: B Baruah; E-mail: binitabaruah17@gmail.com
}

Received: 20 Sept., 2019

Revised: 19 Nov., 2019

Accepted: 26 Nov., 2019

\begin{abstract}
An investigation was carried out during the period from the month of April 2017 to March 2018 to study the non-infectious pathological conditions of liver and kidneys of pigs in and around Guwahati city. A total of 29 cases of non-infectious pathological condition of the liver and kidneys were recorded out of 53 necropsied pigs. The samples were collected for both histopathology as well as for bacterial isolation. Gross and histopathological studies revealed variable histopathological changes such as congestion, haemorrhage, fatty changes and necrosis (Centrilobular and panlobular) in the liver. Kidneys showed haemorrhages, nephrosis, fatty changes, necrosis, atrophy of glomeruli.
\end{abstract}

Keywords: Kidney, Liver, Non-infectious, Pigs

Liver and kidneys are two most important vital organs, that performs varieties of metabolic functions and are prone to various injuries and insults. Liver has detoxifying capacity and detoxifies complex harmful chemical substance to a simple harmless substances through a series of biochemical reactions. During this process free radicals are generated which damages hepatic cells. Due to these important activities liver is commonly exposed to a number of insults for which liver is the first organ to undergo various pathological changes including cellular swelling, fatty change, degeneration and necrosis etc (Vegad, 2007). Similarly, kidneys are the major vital organ of excretion of metabolic wastes so any damage to the kidneys may lead to failure of the kidneys to remove waste products due to which various toxins keep on building up inside the body leading to incompatibility in surviving. Kidneys are also susceptible to a number of insults for which it undergoes some pathological changes including degeneration (Nephrosis), necrosis of renal tubules, inflammatory condition (Nephritis), hydronephrosis etc.

\section{MATERIALS AND METHODS}

A detailed post-mortem examination of 53 carcasses of pigs brought to the Department of Veterinary Pathology, College of Veterinary science, Assam Agricultural University (AAU)), Khanapara, Guwahati during the period April 2017 to March 2018 were carried out. The liver and kidneys were carefully examined for the presence of any pathological changes. The observations were documented in the form of photographs and written reports. For histopathological study, the samples were collected in $10 \%$ neutral buffered formalin. After proper fixation the tissue samples were processed under standard protocol and stained with Hematoxyline and Eosin (H\&E) (Luna, 1968)

Samples of the liver and kidneys were collected aseptically in sterile ziplock bags and kept at $4{ }^{\circ} \mathrm{C}$ in the refrigerator

How to cite this article: Baruah, B., Tamuli, S.M., Rahman, T., Tamuly T. and Gogoi, S. (2019). Pathology of hepato-renal disorder due to non infectious etiology in swine. J. Anim. Res., 9(6): 889-892. 
for bacterial isolation. The samples were streaked onto blood agar or brain heart infusion (BA/BHI) agar plate for primary isolation of bacteria and incubated at $37{ }^{\circ} \mathrm{C}$ for 24-48 hours.

\section{RESULTS AND DISCUSSION}

A detailed PM examination of 53 carcasses of pigs brought to the Department of Veterinary Pathology, C.V. Sc, Khanapara, Guwahati were carried out. A total of 29 cases of non-infectious pathological conditions of liver and kidneys were detected. These samples showed negative results for the presence of bacteria. The cases were classified on the basis of pathomorphological findings. (Table 1).

A total of 29 cases of non-infectious pathological conditions of liver and kidneys were detected. The noninfectious conditions were further classified into hepatic, renal and hepato-renal disorders. There were ten cases of non-infectious pathological conditions of liver. These were further classified into fatty liver, acute hepatic necrosis and cirrhosis.

Six cases of fatty liver were recorded during the present study. Fatty change is most commonly seen in liver and caused by a variety of irritant like hypoxia, hepatotoxins , metabolic diseases like diabetes melitus etc. (Vegad, 2007). Grossly, the liver was pale, greasy and soft in consistency, hepatomegaly. Under microscopic observation, hepatocytes were swollen and rounded. Severe fatty changes including coagulative type of necrosis were markedly evident in microscopic observation. Similar changes were also documented by Vegad (2007), Jones et al. (2006) and Schumacher- Petersen et al. (2019).

A total of four cases of acute hepatic necrosis were recorded during the present study. This condition was most common in young pigs due to Vit E and Se deficiency (Hepatosis dietitica), Cocklebur toxicity, coal tar toxicity, gossypol toxicity. Grossly, the liver showed areas of massive haemorrhages, foci of necrosis and the glisson's capsule was tightly adhered to the liver surface. Microscopically areas of massive pan lobular haemorrhages with centrilobular necrosis which were corroborated with the findings of the previous worker McGavin and Zachary (2012) and Stromberg, (2004).

Five cases of cirrhosis were detected. Cirrhosis is a complication of liver disease involving loss of liver cells and irreversible scarring of the liver. Grossly, the livers were hard and had poor elasticity. Livers were cut with difficulty. Microscopically, the hepatocytes undergo degeneration and necrosis. Proliferation and dilatation of bile ducts were observed and moreover fibrous connective tissues divided the individual hepatic lobules leading to pseudolobulation. Similar lesions of cirrhosis were. reported by Wang et al. (2015) and Rosell (2000).

A total of eleven cases of non-infectious pathological conditions of kidneys were recorded. These were further classified into nephrosis and polycystic kidneys.

Six cases of nephrosis were recorded during the present study. Nephrosis is an acute tubular degeneration leading

Table 1: Diagnosis of postmortem cases on the basis of pathomorphological findings

\begin{tabular}{|c|c|c|c|c|c|c|}
\hline Pathological conditions/diseases & $\begin{array}{l}\text { No of } \\
\text { cases }\end{array}$ & $\begin{array}{c}\text { Percentage of } \\
\text { death }(\%)\end{array}$ & Dysfunctions & Hepatic & Renal & Hepato-Renal \\
\hline (A) Hepatic disorders & 15 & 51.7 & & & & \\
\hline (a) Fatty liver & 6 & 20.7 & Hepatic & ++++++ & - & - \\
\hline (b) Acute hepatic necrosis & 4 & 13.8 & Hepatic & ++++ & - & - \\
\hline (c) Cirrhosis & 5 & 17.2 & Hepatic & +++++ & - & - \\
\hline (B) Renal disorders & 11 & 37.9 & & & & \\
\hline (a) Nephrosis & 6 & 20.7 & Renal & - & ++++++ & - \\
\hline (b) Polycystic kidneys & 5 & 17.2 & Renal & - & +++++ & - \\
\hline (C) Hepato-renal disoders & 3 & 10.3 & & & & \\
\hline (a) Anaemia & 3 & 10.3 & Hepato-renal & - & - & +++ \\
\hline Total & 29 & & & $15(51.7)$ & 11(37.9) & $3(10.3)$ \\
\hline
\end{tabular}

*NB: "+" Indicates number of animals affected by hepatic, renal and hepato-renal dysfunctions. 


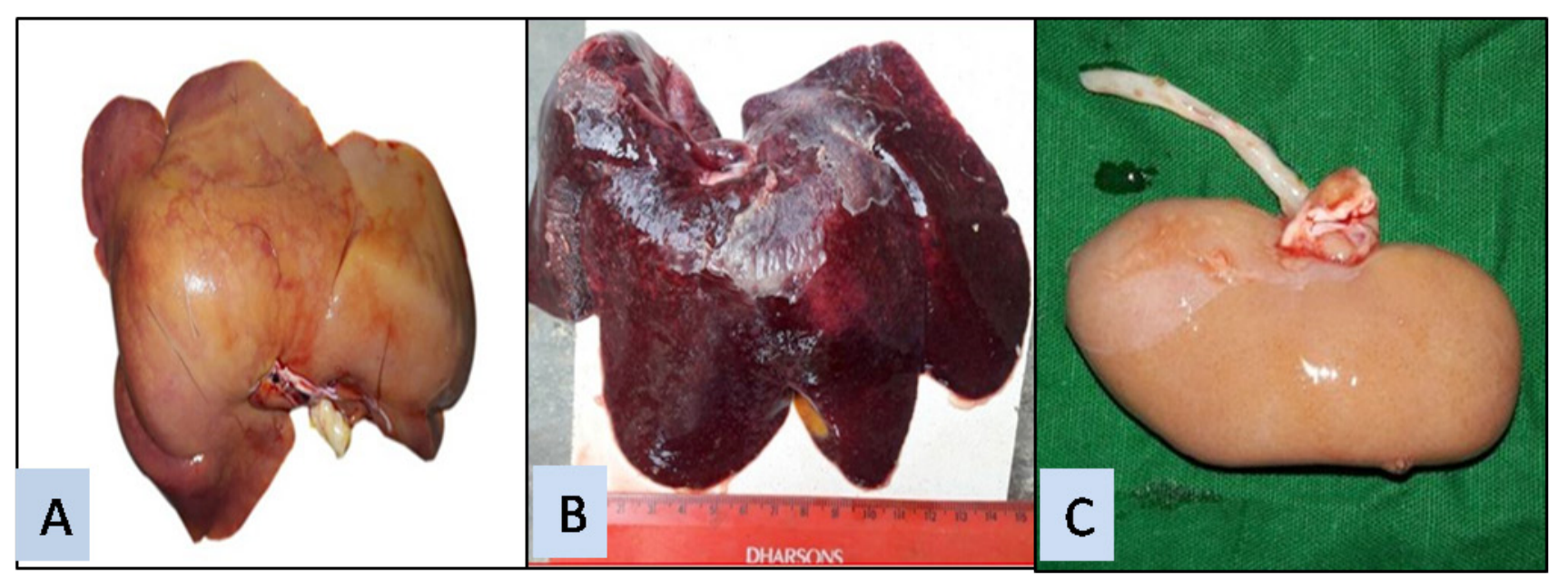

Fig. 1: Macroscopic lesions in the visceral organs observed during post-mortem examination. (A) Fatty liver showing yellowish discolouration of the surface. (B) Liver showing massive haemorrhage, necrosis and adhesion of the glisson's capsule. (C) Kidney showing pale discoloration of the cortex
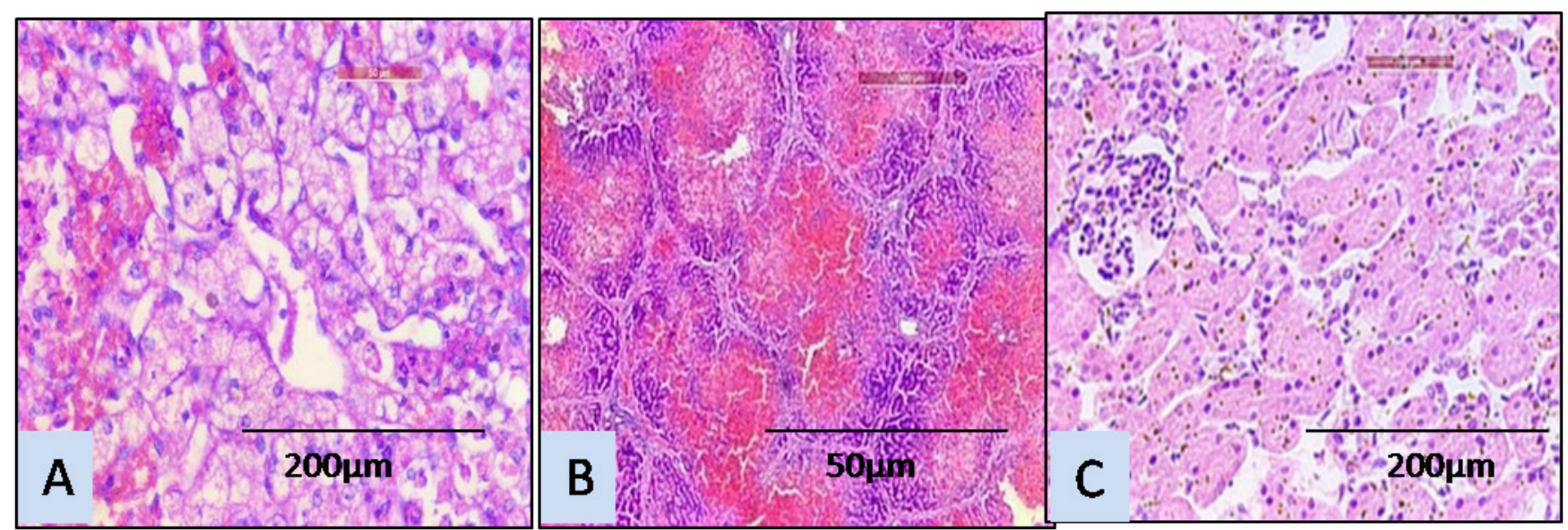

Fig. 2: Microscopic histopathological changes in the visceral organs of (A) showing fatty changes and diffuse hamorrhages within the hepatic parenchyma. (B) showing massive panlobular haemorrhages with severe necrosis of the hepatic parenchyma. (C) showing coagulative necrosis of the renal tubular epithelium

to necrosis. The condition results from ischaemia or toxic damage to the renal tubular epithelial cells. Heavy metals, mercury, $\mathrm{Pb}, \mathrm{Ar}$, antibacterial and antifungal agents, drugs, toxic plants, oxalates were responsible for causing nephrosis (Vegad, 2007) Grossly, the kidneys appeared enlarged and pale. Microscopic observation revealed variable degenerative changes including pyknosis of the nucleus, fatty changes in the tubular epithelium Similar changes were described by Vegad (2007) and Gresham et al. (2006).

A total of five cases of polycystic kidneys were recorded. Grossly, the kidneys were enlarged and soft with thinning of the cortex. On incision multiple cysts with serous fluid were observed. Microscopically, the cystic cavity was emptied surrounded by a serous membrane and fibrous connective tissues. There was atrophy of the renal tubules and glomeruli around the cysts. These findings were in accordance with the findings of McGavin, and Zachary (2012), Kumar et al. (2016) and Adarsh et al. (2010).

A total of three cases of non-infectious pathological conditions affecting both liver and kidneys.

Three cases of anemic piglets were recorded during the present study. Grossly, the carcasses showed pale to 
yellowish discoloration of the mucous membrane of the eyes, hairless part of the skin, visceral organ including liver, kidneys, lungs and intestine. Microscopic lesions in the liver showed centrilobular necrosis, atrophy of the hepatoocytes, separation of hepatic cords and massive dilatation of sinusoidal spaces. Centrilobular regions of hepatic lobules received blood at last i.e., least oxygenated blood and hypoxia develop which eventually results in degeneration and necrosis of hepatocytes, atrophy of hepatocytes and sinusoidal dilatation McGavin and Zachary (2012), Godyn et al. (2016).

\section{REFERENCES}

Adarsh, V., Vijay, A. and Pankaj, P. 2010. Autosomal Dominant Polycystic Kidney Disease: A Comprehensive Review. In. J. Nephrourology, 2: 172-192.

Godyń, D., Pieszka, M., Lipiński, P. and Starzyński, R.R. 2016. Diagnostics of iron deficiency anaemia in piglets in the early postnatal period - a review. Anim. Sci. Papers Report, 34(4): 307-318.

Gresham, A., Done, S., Livesey, C., Macdonald. S., Chan, D., Sayers, R., Clark, C. and Kemp, P. 2006. Survey of pigs' kidneys with lesions consistent with PMWS and PDNS and ochratoxicosis. Part 1: concentrations and prevalence of ochratoxin A. Vet. Rec., 159(22): 737-42.

Jones, T.C., Hunt, R.D. and King, N.W. 2006. Cellular infiltration and degenerations. In: Veterinary Pathology. $6^{\text {th }}$ Edn., Blackwell Publishing, Oxford, U.K., pp. 33-62.
Kumar, K., Gupta, M.K., Singh, K.K. and Kumar, S. 2016. Pathomorphology of Porcine Polycystic Kidney: A Case Report. Ind. J. Vet. Pathol., 40(2): 181-182.

McGavin, M.D. and Zachary, J.F. 2012. Hepatobiliary system and exocrine pancreas. In: Pathologic basis of Veterinary Diseases. $5^{\text {th }}$ Edn., Elseiver, London,U.K., pp. 441-448.

Neumann, E.J., Ramirez, A. and Schwartz, K.J. 2019. Iron Deficiency Anemia. In: Swine Manual Index. Department of Veterinary Diagnostic \& Production Animal Medicine. College of Veterinary Medicine, Iowa State University, pp. 107

Rosell, C., Segales, J. and Domingo, M. 2000. Hepatitis and staging of hepatic damage in pigs naturally infected with porcine circovirus Type 2. Vet. Path., 37: 687-692.

Schumacher, P.C., Christoffersen, O.B., Kirk, K.R., Ludvigsen, P.T., Zois, E.N., Pedersen, D.H., Vyberg, M. and Olsen, H.L. 2019. Experimental non-alcoholic steatohepatitis in Göttingen Minipigs: consequences of high fat-fructose-cholesterol diet and diabetes. J. Trans. Med., 17: 110.

Stromberg, P.C. 2004. The Microscopic Basis for Macroscopic. College of Veterinary Medicine, Ohio State University, pp. 14.

Vegad, J.L. 2007. Cell injury and cell death. In: A textbook of Veterinary General Pathology. $2^{\text {nd }}$ Ed., International Book Distributing Co., U.P. India, pp. 20-21.

Wang, L., He, F.L., Liu, F.Q., Yue, Z.D. and Zhao, H.W. 2015. Establishment of a hepatic cirrhosis and portal hypertension model by hepatic arterial perfusion with $80 \%$ alcohol. World J. Gastroenterol., 21(32): 9544-9553. 